\title{
Diabetes, microvascular complications, and cardiovascular complications: what is it about glucose?
}

\author{
Jane E.B. Reusch \\ Veterans Affairs Medical Center and Division of Endocrinology, Metabolism and \\ Diabetes, Department of Medicine, University of Colorado Health Sciences Center, \\ Denver, Colorado, USA
}

\begin{abstract}
Glycemic control is the primary mediator of diabetic microvascular complications and also contributes to macrovascular complications. A new study (see related article beginning on page 1049) reveals a previously unrecognized association between oxidant activation of poly(ADP ribose) polymerase (PARP) and upregulation of known mediators of glycemic injury. Inhibitors of PARP may have potential therapeutic roles in the prevention of diabetic complications.
\end{abstract}

\section{J. Clin. Invest. 112:986-988 (2003). doi:10.1172/JCI200319902.}

The prevalence of obesity, diabetes, and the metabolic syndrome is increasing in the US and worldwide at astonishing rates $(1,2)$. Diabetes is the leading cause of blindness, renal failure, and amputation in the United States. These conditions can be slowed or prevented with optimal glycemic control $(3,4)$. Accompanying the diabetes epidemic is a concomitant two- to four-fold excess risk for cardiovascular disease $(5,6)$. Modification of traditional cardiovascular risk factors has an impressive impact on morbidity and mortality in subjects with diabetes and insulin resistance as reported in various studies, including the Scandinavian Simvastatin Survival Study $(7,8)$, the Cholesterol and Recurrent Events (CARE) study (9), the MRC/BHF Heart Protection Study (10), the Steno-2 study (11), the Losartan Intervention For Endpoint reduction in hypertension study (12; known as LIFE), and the Heart Outcomes Prevention Evaluation study (13; known as HOPE). Even

\footnotetext{
Address correspondence to: Jane E.B. Reusch, Section of Endocrinology $(111 \mathrm{H})$, Veterans Affairs Medical Center, 1055 Clermont Street, Denver, Colorado 80220, USA. Phone: (303) 399-8020 ext. 2775; Fax: (303) 393-5271;

E-mail: jane.reusch@uchsc.edu.

Conflict of interest: The author has declared that no conflict of interest exists.

Nonstandard abbreviations used: advanced glycation end product (AGE); reactive oxygen species (ROS); poly(ADP-ribose) polymerase (PARP).
}

diabetics, but hyperglycemia adds additional risk.

Strong epidemiological evidence suggests a correlation among glucose, atherosclerotic plaque burden, cardiovascular events, and increased morbidity and mortality (15-17). In an autopsy study of 18- to 34-year-olds there was an increase in atherosclerotic plaque burden in subjects with elevated hemoglobin A1C (17). The Honolulu Heart Program demonstrated a predictive correlation between fasting plasma glucose levels (nondiabetic, impaired glucose tolerance, and diabetic ranges) and cardiovascular events and mortality (18). Large population studies from Northern Europe indicate a direct correlation between glycemic control (as measured by glycohemoglobin) and cardiovascular morbidity and mortality $(16,19)$. As will be discussed later, hyperglycemia has specific deleterious effects upon vascular endothelial function that could account for these epidemiological correlations between hyperglycemia and poor vascular outcomes. One would predict, based upon epidemiological data, that interventional studies targeting hyperglycemia would show improved cardiovascular outcomes. To date, no such compelling evidence has emerged. In fact, in the largest prospective glucose-lowering trial in type 2 diabetes patients, the United Kingdom Prospective Diabetes Study (4), there were no statistical improvements in cardiovascular outcomes when glucose was lowered using insulin or sulfonylureas. Only in the small metformin cohort $(n=342)$ were cardiovascular outcomes improved by optimal glycemic control. A few small studies have suggested a positive impact of glycemic control on cardiovascular events, but this point remains highly debated. The conclusions of a recent panel convened by the American Heart Association suggest that while glycemia contributes to cardiovascular risk, treatment of glycemia, exclusive of other potent cardiovascular risk factor intervention, is inadequate to reverse or reduce the complex atherosclerotic process (18).

\section{Mechanism of glucotoxicity}

The negative impact of hyperglycemia on endothelial function and patho- 


\section{Figure 1}

High glucose flux through constitutive glucose transporters on endothelial cells overwhelms the mitochondrial electron transport system. Excess mitochondrial substrate flux results in the generation of reactive oxygen species that cause DNA strand breaks and activation of PARP. PARP ribosylates and inactivates GAPDH, thereby disrupting normal glucose metabolism. Inactivation of GAPDH effectively shunts glucose into the polyol pathway and leads to activation of PKC and accumulation of AGEs and glucosamine. DAG, diacylglycerol.

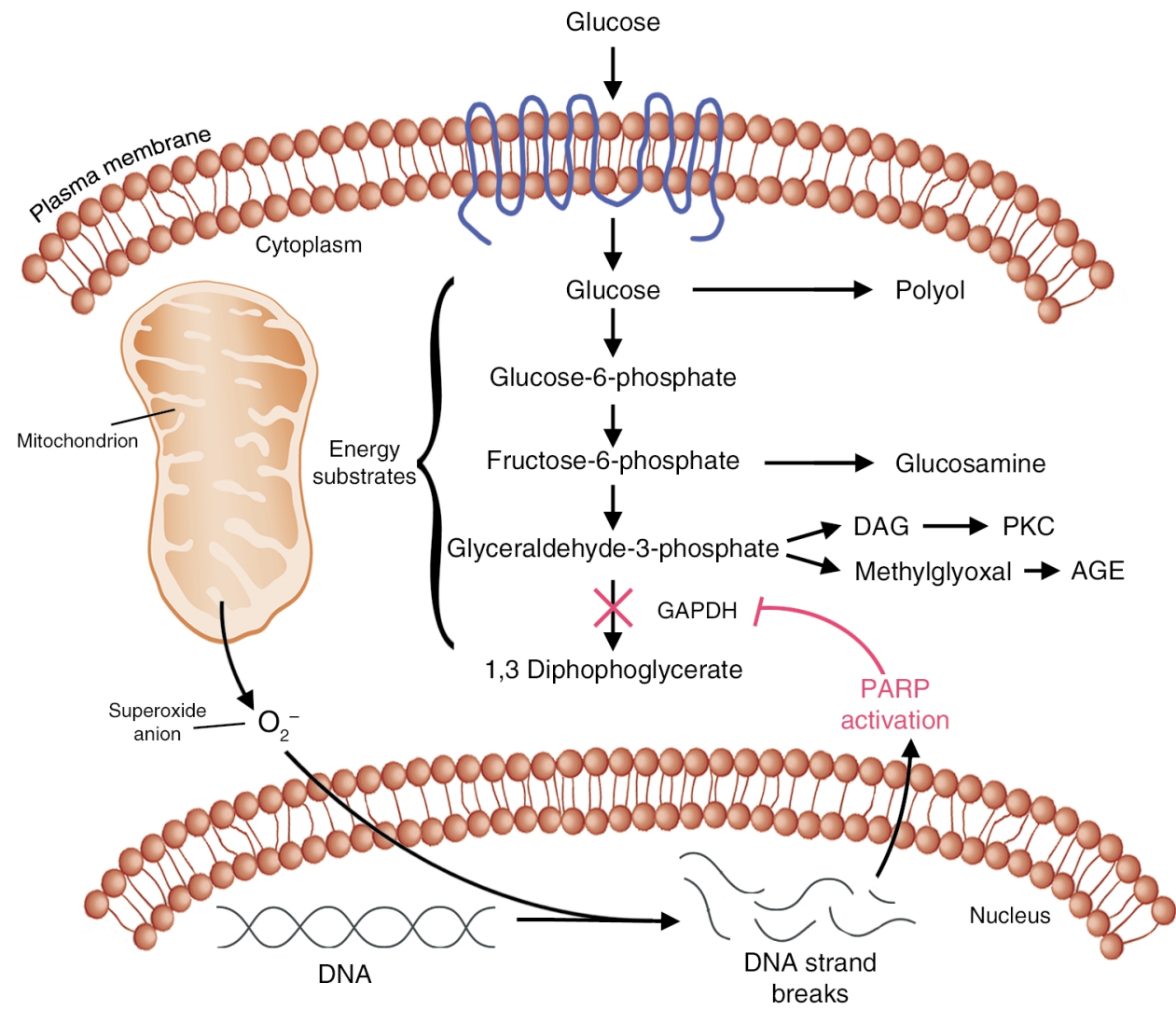

logical changes observed in diabetes is supported in the literature (reviewed in refs. 20,21). Endothelial cells in vitro are exquisitely sensitive to high glucose (25mM). Nishikawa et al. (21) and others have carefully characterized four major molecular signaling mechanisms activated by hyperglycemia in endothelial cells and other cell types vulnerable to hyperglycemic injury. These include activation of PKC (via diacylglycerol), increased hexosamine pathway flux, increased advanced glycation end product (AGE) formation, and increased polyol pathway flux. Nishikawa et al. recently proposed the existence of a unifying mechanism that integrates the above pathways: increased production of reactive oxygen species (ROS) (specifically superoxide) by the mitochondrial electron transport chain (21). In their original report, numerous theoretical constructs were outlined for the impact of altered redox state upon formation of polyols, AGEs, and PKC. What remained unclear were the downstream targets of oxidant stress. In the paper by Du et al. (14) in this issue of the JCI, this group takes their seminal observation one step further and defines one consequence of increased ROS, namely activation of PARP. PARP activation leads to ribosylation and inactivation of GAPDH. Inhibition of GAPDH increases delivery of glycolytic intermediates to the mitochondria. This change in turn would be expected to increase mitochondrial superoxide production and also to increase flux through the AGE and PKC glucotoxic pathways (Figure 1).

\section{Implications for therapy}

It has been well established that diabetes leads to microvascular complications, and it has also been suggested that hyperglycemia plays an accelerating role in macrovascular disease. This excess disease burden is driven by glucose-related activation of PKC, accumulation of AGEs, excess polyol flux, and accumulation of glucosamine (20, 22-25). Interventions for each of these mechanisms have had great efficacy in animal models but disappointing outcomes in clinical trials (26). It was concluded that a cocktail of inhibitors might be necessary to effectively block these deleterious cellular responses in a complex human model of fluctuating hyperglycemia (20). The observation that these pathways reflect a single hyperglycemia-induced process, namely oxidative stress, suggested that antioxidants could serve as a single agent in the prevention of diabetes complications. To date, human studies with $\alpha$ lipoic acid suggest a therapeutic benefit $(27,28)$, but the use of conventional antioxidants has been disappointing in larger trials (29). Conventional antioxidants scavenge free radicals in an inefficient stoichiometric manner so that one molecule of the antioxidant would be needed to neutralize each free radical generated. Novel small molecular weight compounds that function as superoxide dismutase mimetics may offer more reliable benefits due to the catalytic properties that could permit enzymatic detoxification (30). The observation that PARP drives glucotoxicity through inhibition of GAPDH suggests PARP inhibitors as another therapeutic tool for complication prevention. In theory, these agents could have an effect equivalent to combination inhibitor therapy due to positive 
effects on AGE, PKC, and NF- $\mathrm{B}$. Indeed, the few reports employing PARP inhibitors in animal models of diabetes support the therapeutic potential of these agents $(31,32)$. With the incidence of diabetes and its complications on the rise, these results offer hope for new treatments in the foreseeable future.

\section{Acknowledgments}

Jane Reusch is supported by grants from the Veterans Administration Merit Review and Research Enhancement Awards Program, the National Institutes of Health, the Juvenile Diabetes Research Foundation International, the American Heart Association, and the American Diabetes Association.

1. Boyle, J.P., et al. 2001. Projection of diabetes burden through 2050: impact of changing demography and disease prevalence in the U.S. Diabetes Care. 24:1936-1940.

2. Benjamin, S.M., Valdez, R., Geiss, L.S., Rolka, D.B., and Narayan, K.M. 2003. Estimated number of adults with prediabetes in the U.S. in 2000: opportunities for prevention. Diabetes Care. 26:645-649.

3. The Diabetes Control and Complications Trial Research Group. 1993. The effect of intensive treatment of diabetes on the development and progression of long-term complications in insulin-dependent diabetes mellitus. N. Engl. J. Med. 329:977-986.

4. UK Prospective Diabetes Study (UKPDS) Group. 1998. Intensive blood-glucose control with sulphonylureas or insulin compared with conventional treatment and risk of complications in patients with type 2 diabetes (UKPDS 33). Lancet. 352:837-853.

5. Hanson, R.L., Imperatore, G., Bennett, P.H., and Knowler, W.C. 2002. Components of the "metabolic syndrome" and incidence of type 2 diabetes. Diabetes. 51:3120-3127.

6. Abbasi, F., Brown, B.W., Jr., Lamendola, C., McLaughlin, T., and Reaven, G.M. 2002. Relationship between obesity, insulin resistance, and coronary heart disease risk. J. Am. Coll. Cardiol. 40:937-943.

7. Haffner, S.M., et al. 1999. Reduced coronary events in simvastatin-treated patients with coro- nary heart disease and diabetes or impaired fasting glucose levels: subgroup analyses in the Scandinavian Simvastatin Survival Study. Arch. Intern. Med. 159:2661-2667.

8. Pyorala, K., et al. 1997. Cholesterol lowering with simvastatin improves prognosis of diabetic patients with coronary heart disease. A subgroup analysis of the Scandinavian Simvastatin Survival Study (4S). Diabetes Care. 20:614-620.

9. Goldberg, R.B., et al. 1998. Cardiovascular events and their reduction with pravastatin in diabetic and glucose-intolerant myocardial infarction survivors with average cholesterol levels: subgroup analyses in the cholesterol and recurrent events (CARE) trial. The Care Investigators. Circulation. 98:2513-2519.

10. Heart Protection Study Collaborative Group. 2003. MRC/BHF Heart Protection Study of cholesterol-lowering with simvastatin in 5963 people with diabetes: a randomised placebo-controlled trial. Lancet. 361:2005-2016.

11. Pedersen, O., and Gaede, P. 2003. Intensified multifactorial intervention and cardiovascular outcome in type 2 diabetes: The Steno- 2 study. Metabolism. 52:19-23.

12. Lindholm, L.H., et al. 2002. Cardiovascular morbidity and mortality in patients with diabetes in the Losartan Intervention For Endpoint reduction in hypertension study (LIFE): a randomised trial against atenolol. Lancet. 359:1004-1010.

13. Heart Outcomes Prevention Evaluation (HOPE) Study Investigators. 2000. Effects of ramipril on cardiovascular and microvascular outcomes in people with diabetes mellitus: results of the HOPE study and MICRO-HOPE substudy. Lancet. 355:253-259.

14. Du, X., et al. 2003. Inhibition of GAPDH activity by poly(ADP-ribose) polymerase activates three major pathways of hyperglycemic damage in endothelial cells. J. Clin. Invest. 112:1049-1057. doi:10.1172/JCI200318127.

15. Tominaga, M., et al. 1999. Impaired glucose tolerance is a risk factor for cardiovascular disease, but not impaired fasting glucose. The Funagata Diabetes Study. Diabetes Care. 22:920-924.

16. Laakso, M. 1996. Glycemic control and the risk for coronary heart disease in patients with noninsulin-dependent diabetes mellitus. The Finnish studies. Ann. Intern. Med. 124:127-130.

17. Ledru, F., et al. 2001. New diagnostic criteria for diabetes and coronary artery disease: insights from an angiographic study. J. Am. Coll. Cardiol. 37:1543-1550.

18. Donahue, R.P., Abbott, R.D., Reed, D.M., and Yano, K. 1987. Postchallenge glucose concentration and coronary heart disease in men of Japanese ancestry. Honolulu Heart Program. Diabetes. 36:689-692.

19. Kuusisto, J., Mykkanen, L., Pyorala, K., and Laak- so, M. 1994. NIDDM and its metabolic control predict coronary heart disease in elderly subjects. Diabetes. 43:960-967.

20. Sheetz, M.J., and King, G.L. 2002. Molecular understanding of hyperglycemia's adverse effects for diabetic complications. JAMA. 288:2579-2588.

21. Nishikawa, T, et al. 2000. Normalizing mitochondrial superoxide production blocks three pathways of hyperglycaemic damage. Nature. 404:787-790.

22. Schmidt, A.M., et al. 1995. Advanced glycation endproducts interacting with their endothelial receptor induce expression of vascular cell adhesion molecule-1 (VCAM-1) in cultured human endothelial cells and in mice. A potential mechanism for the accelerated vasculopathy of diabetes. J. Clin. Invest. 96:1395-1403.

23. Hart, G.W. 1997. Dynamic O-linked glycosylation of nuclear and cytoskeletal proteins. Annu. Rev. Biochem. 66:315-335.

24. Greene, D.A., Arezzo, J.C., and Brown, M.B. 1999. Effect of aldose reductase inhibition on nerve conduction and morphometry in diabetic neuropathy. Zenarestat Study Group. Neurology. 53:580-591.

25. Vlassara, H., Brownlee, M., Manogue, K.R. Dinarello, C.A., and Pasagian, A. 1988. Cachectin/ TNF and IL-1 induced by glucose-modified proteins: role in normal tissue remodeling. Science. 240:1546-1548.

26. Brownlee, M. 2001. Biochemistry and molecular cell biology of diabetic complications. Nature. 414:813-820.

27. Evans, J.L., and Goldfine, I.D. 2000. Alpha-lipoic acid: a multifunctional antioxidant that improves insulin sensitivity in patients with type 2 diabetes. Diabetes Technol. Ther. 2:401-413.

28. Ametov, A.S., et al. 2003. The sensory symptoms of diabetic polyneuropathy are improved with alpha-lipoic acid: the SYDNEY trial. Diabetes Care. 26:770-776

29. Heart Outcomes Prevention Evaluation Study Investigators. Effects of ramipril on cardiovascular and microvascular outcomes in people with diabetes mellitus: results of the HOPE study and MICRO-HOPE substudy. Lancet. 355:253-259.

30. Ross, A.D., et al. 2002. Hemodynamic effects of metalloporphyrin catalytic antioxidants: structure-activity relationships and species specificity. Free Radic. Biol. Med. 33:1657-1669.

31. Pacher, P., et al. 2002. The role of poly(ADPribose) polymerase activation in the development of myocardial and endothelial dysfunction in diabetes. Diabetes. 51:514-521.

32. Soriano, F.G., Pacher, P., Mabley, J., Liaudet, L., and Szabo, C. 2001. Rapid reversal of the diabetic endothelial dysfunction by pharmacological inhibition of poly(ADP-ribose) polymerase. Circ. Res. 89:684-691. 